\title{
Spatiotemporal dynamics of a free electron maser oscillator with broadband feedback and klystronlike interaction region
}

\author{
A. V. Savilov* and N. Yu. Peskov \\ Institute of Applied Physics, Russian Academy of Sciences, 46 Ulyanov Street, Nizhny Novgorod, 603950, Russian Federation \\ V. N. Philippov \\ Nizhny Novgorod State University, Gagarina Avenue 23, Nizhny Novgorod, 603950, Russian Federation
}

(Received 18 January 2005; published 31 August 2005)

\begin{abstract}
Dynamics of interaction of longitudinal modes in a FEM oscillator with a broad-frequency-band feedback system and a klystronlike interaction region is studied. It is shown that the use of the klystronlike interaction region results both in increasing the efficiency of electron energy extraction and decreasing the number of competing modes. The latter provides a significant increase of the electron-current threshold of stability of the single-mode operation.
\end{abstract}

DOI: $10.1103 /$ PhysRevSTAB.8.080702

PACS numbers: $84.40 . \mathrm{Fe}, 41.60 . \mathrm{Cr}$

\section{INTRODUCTION}

The use of a klystronlike interaction region is a wellknown method for improving the operation of free-electron lasers and masers (FELs and FEMs) (see, e.g., [1-3]). A principal schematic of a klystronlike interaction region is shown in Fig. 1. Electrons interact with the operating wave only in the first section $\left(0 \leq Z \leq L_{1}\right.$, where $Z$ is the longitudinal coordinate in any normalization), where the electron beam obtains initial energy modulation, and in the third section $\left(L_{2} \leq Z \leq L\right)$, where effective extraction of the electron energy takes place. The second section $\left(L_{1} \leq\right.$ $Z \leq L_{2}$ ), where there is no the electron-wave interaction, is needed to provide electron bunching. In FEM ubitrons such a situation can be provided by using a sectioned undulator. We can propose another possibility to provide a klystronlike interaction region. This is the use of a complicated operating waveguide, where the operating wave is transformed at the border between the first and the second sections into an auxiliary wave, which does not interact with electrons; at the border between the second and the third sections the opposite transformation occurs (Fig. 2).

In this work, we consider the case of a FEM oscillator with a feedback system having a broad frequency band (which can be useful, for instance, in order to provide frequency tuning). In this situation, it is not evident in advance whether the use of a klystronlike interaction region provides efficiency enhancement. Actually, in this case the frequency of the operating wave (the operating longitudinal mode) is not fixed by the microwave system. A large number of longitudinal eigenmodes of the system (of the order of $\gamma^{2}$, where $\gamma$ is the electron Lorentz factor) usually exists in the amplification band, and all these modes arise from initial noises at the small-signal stage of excitation. Then, at the nonlinear stage of the transient

\footnotetext{
*Corresponding author.

Email address: savilov@appl.sci-nnov.ru
}

process, they take part in a complicated process of competition. The result of this process and, correspondingly, the spectrum of the operating (steady-state) regime are determined only by the electron-wave interaction; namely, by the value of the electron current [4-6]. If the current is close to the starting value, then the single-mode steadystate regime is achieved; the efficiency in this regime is determined by the frequency of the excited mode. However, if the current exceeds some threshold, then the single-mode operation is not stable, and the final stage represents the operation of a number of longitudinal modes.

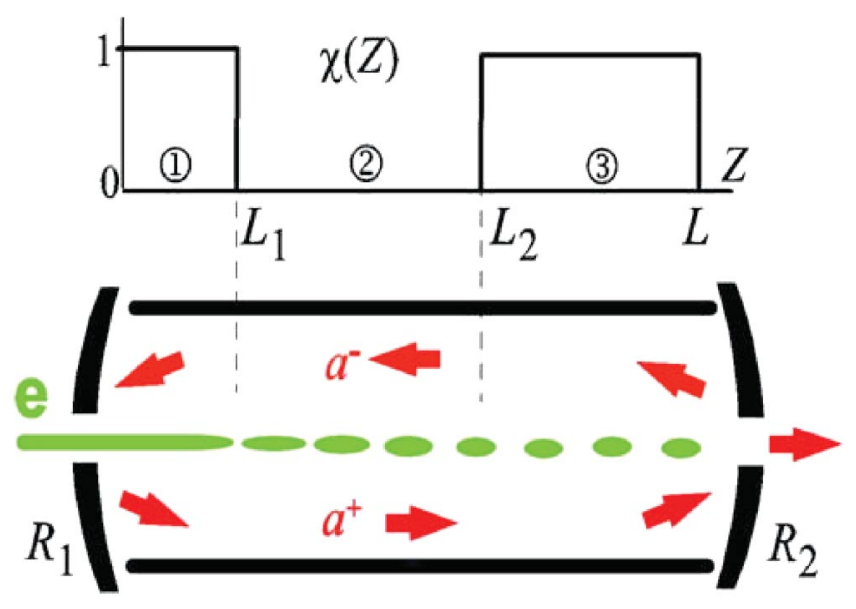

FIG. 1. (Color) Scheme of a FEM oscillator with klystronlike electron-wave interaction region, which consists of regions of electron energy modulation (1), of electron bunching (2), and of electron energy extraction (3). Microwave system (red arrows indicate motion of the operating forward wave and the feedback backward wave) and profile of the electron-wave coupling coefficient $\chi(Z)$. 


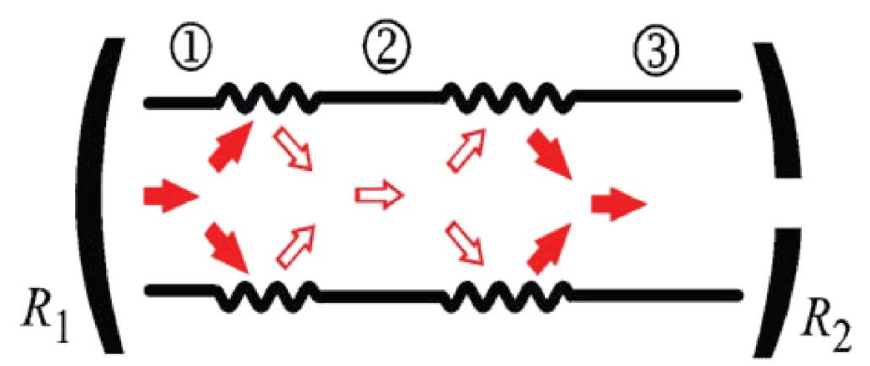

FIG. 2. (Color) Possible variant of realization of the three-stage profiling of the electron-wave coupling, when the operating wave (filled red arrows) is transformed into an auxiliary wave in the bunching region (open red arrows).

\section{SPATIOTEMPORAL APPROACH}

We consider a FEL oscillator with the microwave system consisting of a waveguide segment with two (input and output) frequency-broadband short mirrors (Fig. 1). Let us suppose that the undulator imparts the following transverse motion of electrons,

$$
\mathrm{v}_{x}=\mathrm{v}_{\perp} \cos \left(k_{\mathrm{u}} z\right),
$$

and the particles interact with a forward wave, which field is described by the vector potential

$$
\mathbf{A}^{+}=\operatorname{Re} A^{+}(t, z) \mathbf{f} e^{i \omega_{\mathrm{r}} t-i k_{\| r} z},
$$

where $\omega_{\mathrm{r}}=\mathrm{v}_{\mathrm{e}} h_{\mathrm{r}}=\mathrm{v}_{\mathrm{e}}\left(k_{\| \mathrm{r}}+k_{\mathrm{u}}\right)$ is the resonant frequency, $k_{\| \mathrm{r}}=k_{\|}\left(\omega_{\mathrm{r}}\right)$, and $\mathrm{v}_{\mathrm{e}}$ is the averaged longitudinal velocity of particles unperturbed by the electron-wave energy exchange. As for vector $\mathbf{f}(x, y)$, it describes the transverse structure of the wave is normalized so that $f_{\mathrm{x}}\left(x_{\mathrm{e}}, y_{\mathrm{e}}\right)=1$, where $\left(x_{\mathrm{e}}, y_{\mathrm{e}}\right)$ are unperturbed electron-beam transverse coordinates.

For a number of important cases the dynamics of such an oscillator can be studied successfully within the framework of the spatiotemporal approach based on the simplest 1D nonstationary equations [4-6] for complex amplitudes of forward $\left(a^{+}\right)$and backward $\left(a^{-}\right)$waves,

$$
\frac{\partial a^{+}}{\partial Z}+\frac{\partial a^{+}}{\partial \tau}=2 i \rho, \quad \rho=\chi(Z)\left\langle e^{-i \theta}\right\rangle_{\varphi}, \quad \frac{\partial a^{-}}{\partial Z}-\varepsilon \frac{\partial a^{-}}{\partial \tau}=0,
$$

and motion equations for the electrons coupling with the operating forward wave,

$$
\frac{\partial u}{\partial Z}=\chi(Z) \operatorname{Im} a^{+} e^{i \theta}, \quad \frac{\partial \theta}{\partial Z}=u .
$$

Here $u=\mu C^{-1}\left(\gamma_{0}-\gamma\right) / \gamma_{0}$ is the normalized change in electron energy $(\gamma$ is the relativistic electron Lorentz factor, $\gamma_{0}$ is its initial value), $Z=h_{\mathrm{r}} C z$ is the normalized coordinate, $\theta=\omega_{\mathrm{r}} t-h_{\mathrm{r}} z$ is the electron phase with respect to the resonant combination wave, $\tau=h_{\mathrm{r}} C\left(\mathrm{v}_{\mathrm{e}} t-z\right) /\left(\mathrm{v}_{\mathrm{e}} / \mathrm{v}_{\mathrm{gr}}-1\right)$ is the normalized time, $a^{ \pm}=\left(e k_{\mathrm{r}} / m c^{2} k_{\| \mathrm{r}}\right) \cdot(C / \mu \kappa) A^{ \pm}, \quad \varepsilon=\left(\mathrm{v}_{\mathrm{e}}+\mathrm{v}_{\mathrm{gr}}\right) /\left(\mathrm{v}_{\mathrm{e}}-\right.$ $\left.\mathrm{v}_{\mathrm{gr}}\right)$ is the so-called slippage parameter, $\langle\ldots\rangle_{\varphi}$ denotes averaging over the whole electron ensemble, $v_{\mathrm{gr}}$ is the group wave velocity at the frequency $\omega_{\mathrm{r}}, C=\sqrt[3]{\mu \kappa^{2} G}$ is the Pierce parameter, $G=\left(I / I_{\mathrm{A}}\right) \cdot\left(2 \pi k_{\mathrm{r}} / k_{\| \mathrm{r}}^{3} N\right)$ is the excitation factor, $I$ is the electron current, $I_{\mathrm{A}}=m c^{3} / e \approx$ $17 \mathrm{kA}, N=\iint\left(\left|f_{\mathrm{x}}\right|^{2}+\left|f_{\mathrm{y}}\right|^{2}\right) d x d y$ is the wave norm, $\mu=$ $\gamma_{0}^{-2}$ and $\kappa=v_{\perp} / 2 \gamma_{0} \mathrm{v}_{\mathrm{e}}$ are the parameters of the inertial bunching of particles and the coefficient of the electronwave coupling, respectively, and $k_{\mathrm{r}}=\omega_{\mathrm{r}} / c$.

In Eqs. (1) and (2), factor $\chi(Z)$ describes the following three-stage profiling of the electron-wave interaction (Fig. 1):

$$
\chi(Z)= \begin{cases}1, & 0<Z<L_{1} \\ 0, & L_{1}<Z<L_{2} . \\ 1, & L_{2}<Z<L\end{cases}
$$

In FEM ubitrons such profiling can be provided by using a sectioned undulator. We can propose another possibility to provide a klystronlike interaction. This is the use of a complicated operating waveguide, where the operating wave is transformed at the border between the first and the second sections into an auxiliary wave, which does not interact with electrons; at the border between the second and the third sections the opposite transformation occurs (Fig. 2). As an example of such a system, we can consider an operating waveguide with a circular cross section and Bragg-type wave transformers, which represent pieces of corrugated waveguides. A corrugation of the wall, $R(z, \varphi)=R_{0}+R_{\mathrm{br}} \cos \left(h_{\mathrm{br}} z+m_{\mathrm{br}} \varphi\right)$, provides mutual coupling of two waves, if parameters of these waves satisfy the Bragg resonant condition:

$$
\left|k_{|| 1}-k_{|| 2}\right|=h_{\mathrm{br}}, \quad\left|m_{1}-m_{2}\right|=m_{\mathrm{br}},
$$

where $k_{\| 1,2}$ and $m_{1,2}$ are longitudinal wave numbers and azimuthal indexes of the waves [7]. Let is consider a case, when electrons move along a helical axis-encircling trajectory in the field of a helical undulator. In this case, electrons can interact at the fundamental bounce harmonic only with circularly polarized modes of the operating waveguide, which azimuthal index is equal to unit (for example, $\left.\mathrm{TE}_{1,1}\right)$. Such an interaction is also described by Eqs. (1) and (2), but the electron-wave coupling factor should be twice bigger, $\kappa=v_{\perp} / \gamma_{0} \mathrm{v}_{\mathrm{e}}$. In this case, a helical corrugation of the waveguide wall $\left(m_{\mathrm{br}}=1\right)$ can be used for transformation of this operating wave into the auxiliary wave with a higher azimuthal index (for example, $\mathrm{TE}_{2,1}$ ), which does not interact with the electron beam.

The initial conditions for the unmodulated electron beam at the input of the interaction region, $Z=0$, can be written in the following way:

$$
u(\tau, Z=0)=0, \quad \theta(\tau, Z=0)=\varphi,
$$

where the initial phases, $\varphi$, are distributed homogeneously over the interval $[0,2 \pi]$. 
The simplest boundary condition for the rf field describes the reflection of the waves from the input and the output mirrors:

$$
\begin{aligned}
a^{+}(\tau, Z=0) & =R_{1} a^{-}(\tau, Z=0), \\
a^{-}(\tau, Z=L) & =R_{2} a^{+}(\tau, Z=L) .
\end{aligned}
$$

Here $R_{1,2}$ are the amplitude reflection coefficients of the input and the output mirrors (Fig. 1), which are independent of the frequency.

The system of Eqs. (1)-(4) represents a self-consistent problem, which describes excitation of the FEM oscillator with a nonfixed spatiotemporal structure of the rf filed. It can be solved numerically using standard routines, which are based on the method of cubic Hermite polynomials [8]. In the operating (steady-state) regime, the normalized output $\mathrm{rf}$ power (the normalized efficiency) is determined as follows:

$$
\eta=\langle u\rangle_{\varphi}
$$

The time dependence of the rf amplitudes describes the frequency spectrum of excited longitudinal modes:

$$
a^{ \pm}(\tau)=\int a_{\Delta}^{ \pm} e^{i \Delta \tau} d \Delta
$$

Here the mismatch of the mode $\Delta=\left[\left(\omega-\omega_{\mathrm{r}}\right) / \omega_{\mathrm{r}}\right](1-$ $\left.\mathrm{v}_{\mathrm{gr}} / \mathrm{v}_{\mathrm{e}}\right) C^{-1}$ is the normalized difference between the frequency of this mode and the resonant frequency.

\section{MODE INTERACTION IN A HOMOGENEOUS SYSTEM}

The set of equations (1)-(4) was studied in detail in works $[5,6,9,10]$ for the case of a homogeneous interaction region, $\chi(Z) \equiv 1$. In the case of a fixed feedback factor, $R=R_{1} R_{2}$, the type of the operating regime is determined by parameter $L$, which is proportional to the length of the interaction region, $l$, and to the cubic root from the electron current, $L \propto l \sqrt[3]{I}$. The oscillator is excited if $L$ is larger than the starting value, $L_{\mathrm{st}}(R)$. If parameter $L$ does not exceed the starting value significantly, then the spectrum of the operating regime contains only one excited mode, which has the greatest small-signal single-pass gain (the "main" mode). However, when $L$ exceeds some threshold, $L_{\mathrm{cr}}(R)$, the operation regime has the multimode character.

As an example, we study here the case of a moderately low- $Q$ cavity, $R=0.5$. For this case, $L_{\mathrm{st}} \approx 2.0$ and $L_{\mathrm{cr}} \approx$ 2.7. Figure 3 illustrates dynamics of excitation of the oscillator in the cases of the single-frequency $(L=2.4)$ and multifrequency $(L=3.2)$ steady-state regimes. The dependence of the normalized efficiency, $\eta$, on parameter $L$ in the steady-state regime is shown in Fig. 4.
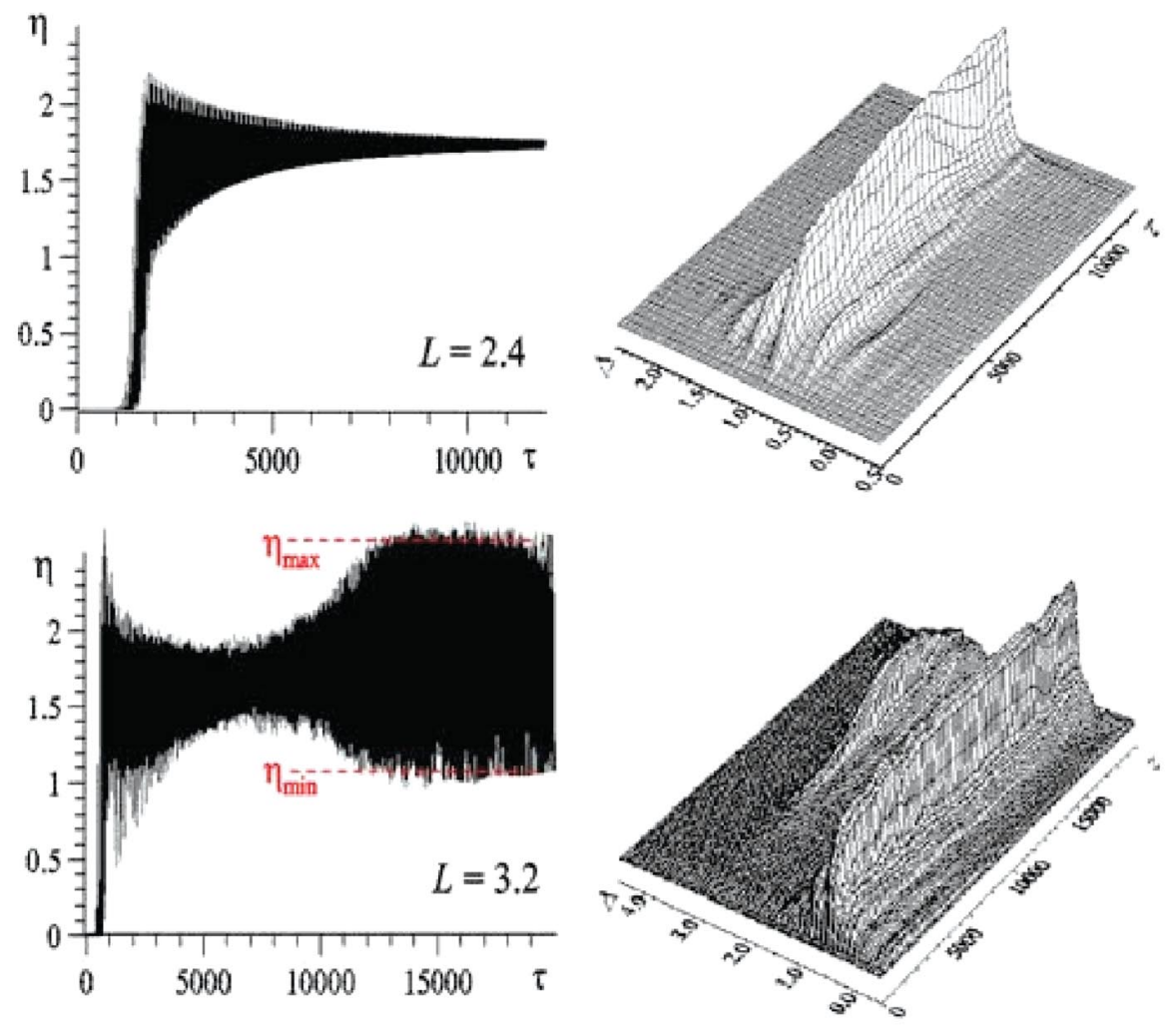

FIG. 3. (Color) Spatiotemporal dynamics of excitation of a FEM oscillator with a homogeneous interaction region $(R=0.5)$. Normalized electronic efficiency, $\eta$, and frequency spectrum of excited modes, $a_{\Delta}$, versus the normalized time, $\tau$, for various lengths of the interaction region, $L$. 


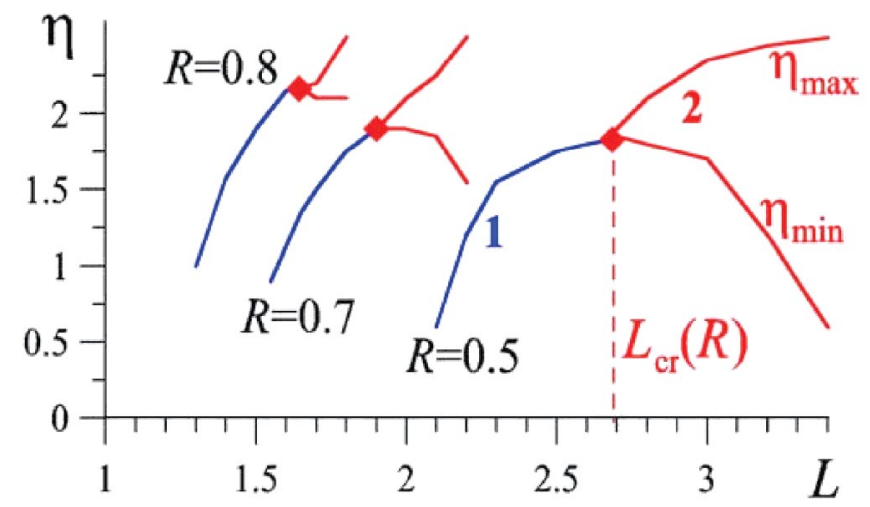

FIG. 4. (Color) Homogeneous interaction region. Dependence of the normalized efficiency in the steady-state regime, $\eta$, on normalized interaction length, $L$, for various feedback factors, $R$. Zones of single-mode (1, blue curve) and multimode (2, red curves) operation regimes are bordered by the critical value of parameter $L$.

In the zone of the stable single-frequency generation $L_{\text {st }}<L<L_{\text {cr }}$ (the blue zone "1" in Fig. 4), the dependence $\eta(L)$ monotonically increases with $L$. The highest efficiency in the single-mode regime is achieved at $L \approx$ $L_{\mathrm{cr}}$ and amounts to $\eta \approx 1.8$. This efficiency is determined by the mismatch of the "main" mode, $\Delta_{\text {main }}$, which corresponds to the maximum of the small-signal single-pass gain, $G(\Delta)=\left|a^{+}(L) / a^{+}(0)\right|$ (Fig. 5). At the linear (smallsignal) stage of the excitation, when all modes of the amplification band $G(\Delta)>R^{-1}$ grow independently, the main mode growths at the greatest rate [Fig. 6(a)]. Thus, this is the first mode achieving the nonlinear stage of the excitation. Because of this fact, at the nonlinear stage the main mode suppresses all other modes [Fig. 6(b)], and, therefore, the single-frequency steady-state regime represents the generation of this mode. Let us note that in this regime, when $\Delta=\Delta_{\text {main }}$, the efficiency is considerably lower than the efficiency optimized over mismatches of all modes, $\Delta$ (Fig. 5).

When $L>L_{\mathrm{cr}}$, the steady-state regime has the multimode character. In this case a fast temporal modulation of the output power occurs due to "beating" of excited modes; this situation is illustrated in Fig. 4 (red zone " 2 ") by two curves of the function $\eta(L)$ corresponding to the lower and upper limits ( $\eta_{\min }$ and $\eta_{\max }$ ) of "beating" of the normalized output rf power (Fig. 3, $L=3.2$ ). The difference between $\eta_{\min }$ and $\eta_{\max }$ increases with the increase of $L$, which corresponds to complicating of the spectrum of modes excited in the built-up regime.

A scenario of transition from the single-mode built-up regime to the multimode one was proposed in $[9,10]$; this scenario is illustrated in Fig. 6. The beginning of the process of the FEM-oscillator excitation is the small-signal stage (a), when all modes of the amplification band $G(\Delta)>R^{-1}$ grow independently. The mode interaction represents a nonlinear process, and it begins at stage (b),

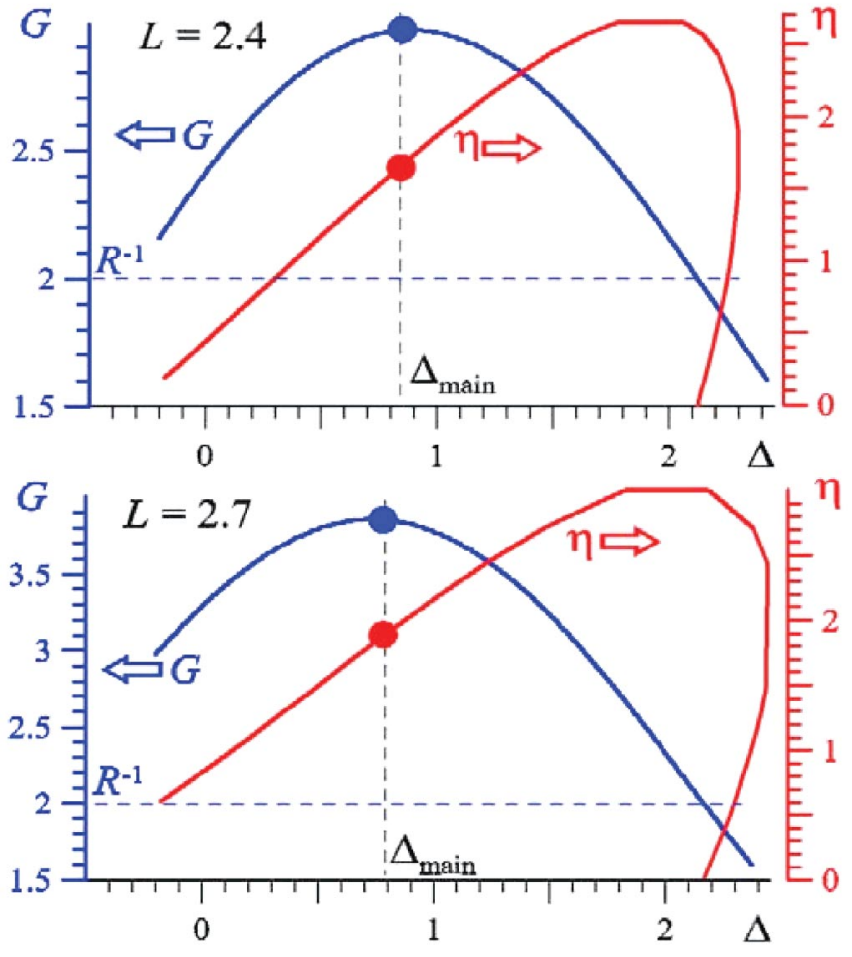

FIG. 5. (Color) Homogeneous interaction region $(R=0.5)$. Small-signal gain, $G$, and efficiency in single-mode regime of generation, $\eta$, versus the mismatch (normalized frequency) of the longitudinal mode, $\Delta$ in the cases of $L=2.4$ and $L=2.7$. Mismatch $\Delta_{\text {main }}$ corresponds to the main mode, which is excited in the case of nonfixed frequency.
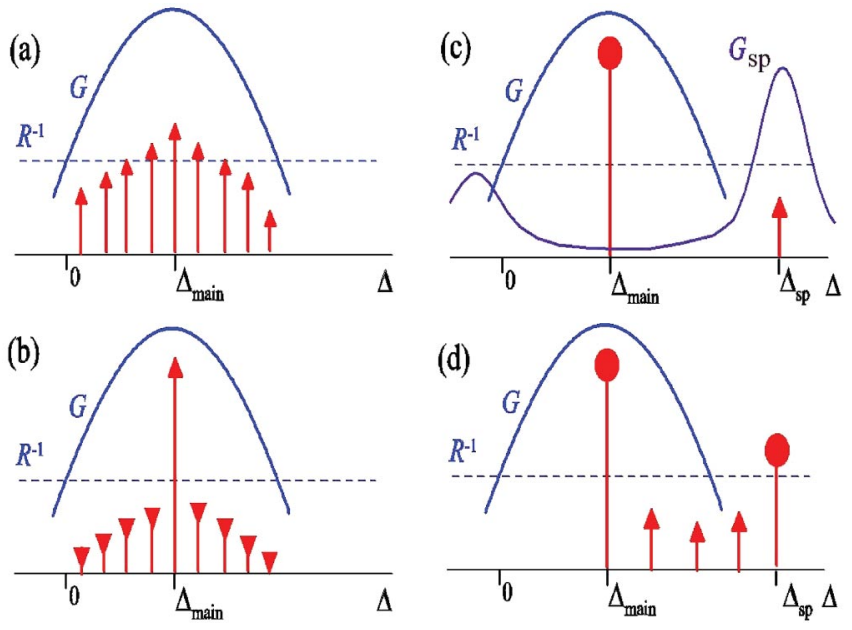

FIG. 6. (Color) Dynamics of the mode competition in a FEM oscillator with a homogeneous interaction region. At the smallsignal stage (a) all modes of the amplification band $G(\Delta)>R^{-1}$ grow independently. At stage (b) the main mode suppresses all other modes of the amplification band. Stages (c) and (d) illustrate the transition to multimode regimes. In this figure, blue curve $G(\Delta)$ is the gain of a mode in the small-signal regime, whereas violet curve $G_{\mathrm{sp}}(\Delta)$ illustrates the gain of spurious modes in the regime of the single-frequency generation of the main mode. 
when the main mode, which grows at stage (a) with the greatest rate, achieves the nonlinear level of the power and suppresses all other modes. If the single-frequency generation of this mode is stable, stage (b) is the last stage of the mode interaction process.

The mode interaction in this system possesses a very important property. Namely, the main mode effectively suppresses only modes having frequencies $\Delta$ quite close to the main mode frequency, $\Delta_{\text {main }}$; this suppression becomes weaker with the increase of the difference $\mid \Delta-$ $\Delta_{\text {main }} \mid$. This effect is illustrated in Fig. 6(c) by the violet curve $G_{\mathrm{sp}}(\Delta)$, which represents the single-pass gain of spurious modes in the regime of the quasistable singlefrequency operation of the main mode [10]. Thus, the single-frequency operation of the main mode can be disturbed only by spurious excitation of "dangerous" modes, the frequencies of which, $\Delta_{\mathrm{sp}}$, are quite far from $\Delta_{\text {main }}$. Let us note that these dangerous spurious modes are not excited at the small-signal stage (a), because they are out of the small-signal amplification band $G(\Delta)>R^{-1}$. However, they can be excited at stage (c), if the following condition is fulfilled:

$$
G_{\mathrm{sp}}\left(\Delta_{\mathrm{sp}}\right)>R^{-1}
$$

In Fig. $3(L=3.2)$ one can clearly see the beginning of stage (c) at $\tau \approx 7000$, when the spurious mode with the normalized frequency $\Delta_{\mathrm{sp}} \approx 2.5$ is excited against a background of the almost single-frequency generation of the main mode, $\Delta_{\text {main }} \approx 0.8$. The appearance of the second mode at stage (c) "spoils" the generation of the main mode and, therefore, weakens the suppression of the other spurious modes. As a result, stage (c) can go on to stage (d), which represents generation of several modes (Fig. 6).

Thus, the key point in losing stability of the single-mode generation is the transition from stage (b) to stage (c); in other words, the excitation of the dangerous spurious mode $\left(\Delta=\Delta_{\mathrm{sp}}\right)$ against a background of the almost singlefrequency generation of the main mode $\left(\Delta=\Delta_{\text {main }}\right)$. Let us notice again that the mismatch of the dangerous spurious mode, $\Delta_{\mathrm{sp}}$, is big enough, so that at the small-signal stage (a) this mode is far from the resonance with electrons, and, therefore, it is not excited. However, at stage (c) this mode can be excited due to the presence of the generation of the main mode. Namely, this mode extracts the electron energy, and if the efficiency of this extraction is great enough, then a part of "spent" electrons become in resonance with the spurious wave. Evidently, condition (5) is satisfied if the normalized efficiency of interaction between electrons and the main mode is as great as the difference in normalized mismatches of the two modes, $\eta \approx \Delta_{\mathrm{sp}}-\Delta_{\text {main }}$. According to [10], in a wide range of the feedback coefficient, $R$, this difference is about 2 .

Thus, in the uniform system there is a limitation of the efficiency of the single-mode operation,

$$
\eta_{\lim } \approx 2 \text {, }
$$

which arises due to transition to the multimode generation at higher efficiencies (Fig. 4). It is demonstrated further that this limit can be significantly increased in the klystronlike scheme of the electron-wave interaction region.

\section{MODE INTERACTION AND EFFICIENCY IN THE KLYSTRONLIKE SCHEME}

As an example, we consider the case of a short first section, $L_{1}=0.2$, and of the feedback factor $R=0.5$. Figure 7 illustrates the dependencies of the normalized efficiency in the steady-state regime, $\eta$, on the total cavity length $L$ at various lengths of the second (bunching) section, $L_{2}$. It is seen that the character of dependencies $\eta(L)$ is similar to the case of a homogeneous interaction region (Fig. 4), namely, stable single-mode regimes at small enough values of parameter $L$, and the multimode regime at big values of $L$. However, the maximal efficiency in the single-mode regime amounts to $\eta \approx 3.7$, which is twice higher than in the case of a homogeneous system.

There is another important peculiarity of the klystronlike system. Namely, curves $\eta(L)$ in Fig. 7 , which describe single-mode regimes, consist of two parts ("1a" and " $1 \mathrm{~b}$ "), which correspond to different types of the modes excited in these regimes. This reflects the fact that in the case of the klystronlike system the amplification band, $G(\Delta)$, has two maximums and, therefore, consists of two separate groups of modes (Fig. 8). This fact can be understood from Fig. 9. In the first section of the interaction region $\left(0 \leq Z \leq L_{1}\right)$ electrons are mixed over their phases

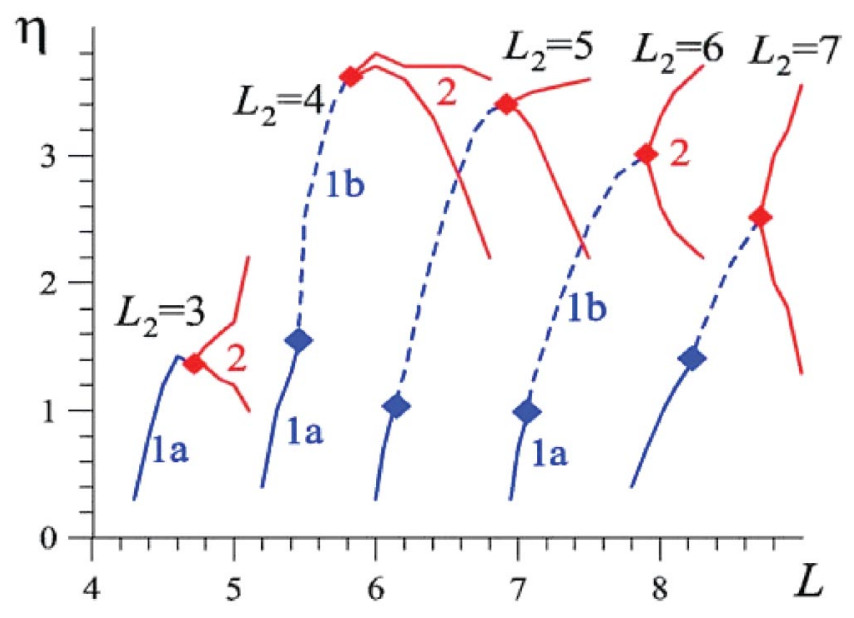

FIG. 7. (Color) Klystronlike interaction region $(R=0.5)$. Normalized efficiency in the steady-state regime, $\eta$, versus on the normalized total cavity length, $L$, at various lengths of the bunching section, $L_{2}$. In this figure, 1 a (solid blue curves) and $1 \mathrm{~b}$ (solid blue curves) denote zones of the stable single-mode generation of a close-to-resonance mode a and a far-fromresonance mode $b$, whereas 2 (red curves) denotes zones of multimode generation. 


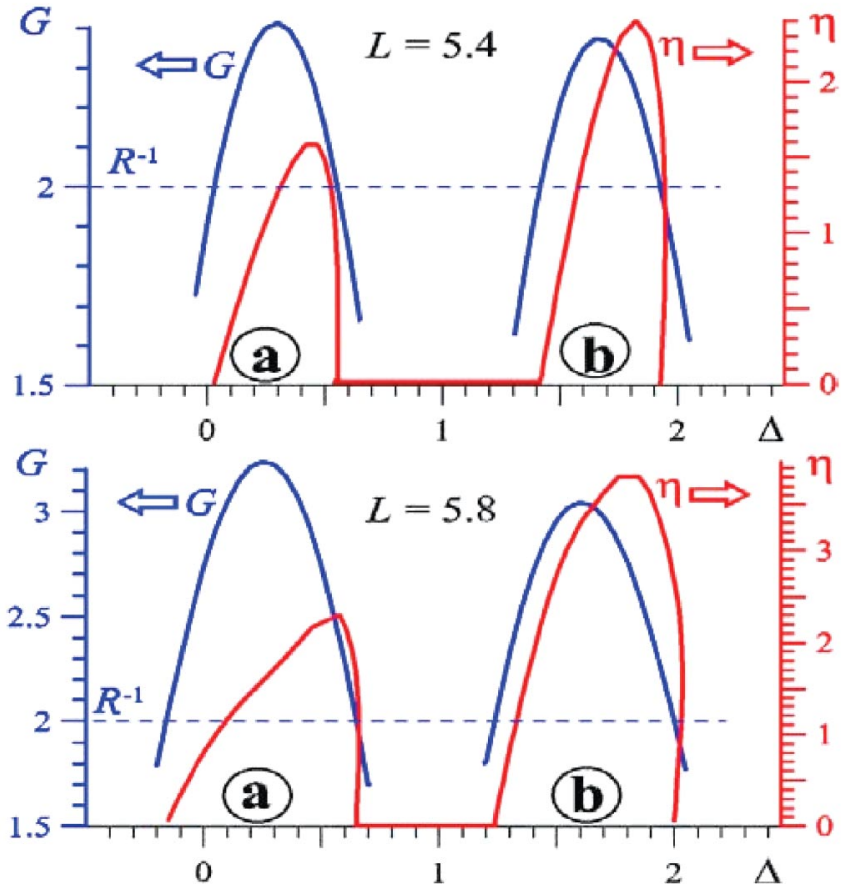

FIG. 8. (Color) Klystronlike interaction region $(R=0.5)$. Small-signal gain, $G$, and efficiency in the single-mode regime of generation, $\eta$, versus the mismatch (normalized frequency) of the longitudinal mode, $\Delta$ in the cases of $L=5.4$ and $L=5.8$ ( $L_{2}=4$ for the both cases). Two groups of excited modes, namely, close-to-resonance a modes and far-from-resonance b modes, are shown.

with respect to the operating wave; namely, a half of the electrons is placed in the accelerating phases of the waves, whereas the other half is placed in the decelerating phases. Because of modulation in electron velocity, in the second section $\left(L_{1}<Z<L_{2}\right)$ electron bunching occurs. In the beginning of this section, the phase of the bunch center

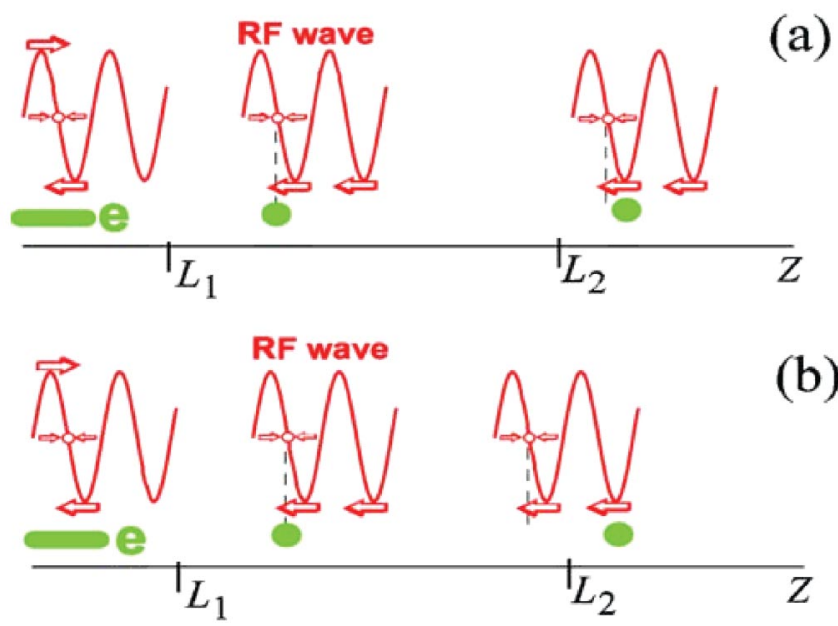

FIG. 9. (Color) Motion of the electron bunch with respect to the combination wave in the case of a close-to-resonance a mode (a) and of a far-from-resonance b mode (b). corresponds to the "zero" of the effective rf wave field. However, in order to provide effective radiation in the third section $\left(L_{2}<Z<L\right)$, the bunch should shift to the maximum of the decelerating rf-wave phase. For the first part of the amplification band ("a" modes in Fig. 8), the bunch shifts from the zero of the wave field to the nearest decelerating maximum [Fig. 9(a)], so that mismatches of these modes, $\Delta_{\mathrm{a}}$, are determined by the following formula:

$$
\Delta_{\mathrm{a}} L_{2} \approx \pi / 2
$$

As for the second part of the amplification band ("b" modes in Fig. 8), it corresponds to the case, when the bunch shifts not to the nearest maximum of the decelerating rf-wave phase, but to the next one [Fig. 9(b)]. Correspondingly, the mismatches of these modes, $\Delta_{\mathrm{b}}$, are bigger:

$$
\Delta_{\mathrm{b}} L_{2} \approx \pi / 2+2 \pi \text {. }
$$

As is seen from Fig. 8, a modes have slightly higher small-signal gains, $G$, whereas b modes have significantly higher efficiencies of electron energy extraction in the nonlinear stage of the interaction, $\eta$. Higher efficiencies in $b$ regimes can be explained easy, if one considers the motion of the electron bunch in the third section $\left(L_{2}<Z<\right.$ $L$ ) using the approximation of a homogeneous rf-wave amplitude, $a(Z, \tau)=a_{0} e^{i \Delta \tau-i \Delta Z}$. According to motion equations (2), in this case the electron motion can be described by the following Hamiltonian:

$$
H=\cos \hat{\theta}+(u-\Delta)^{2} / 2,
$$

where $\hat{\theta}=\theta-\Delta Z$. On the phase plane $(u, \hat{\theta})$ the electron bunch moves along a curve $H=$ const inside the "bucket" (separatrix) described by the following formula:

$$
u(\hat{\theta})=\Delta \pm \sqrt{2(1-\cos \hat{\theta})} .
$$

It is evident from Fig. 10 that in order to obtain a bigger change in electron energy, $u$, one should provide a big enough mismatch, $\Delta$. This is the reason for higher electron efficiencies obtained in $b$ regimes.

In the klystronlike system studied here, the process of mode interaction represents basically a competition between the two groups of modes described above. If the length of the bunching region, $L_{2}$, is short enough, then mismatches of b modes, $\Delta_{\mathrm{b}}$, are too big, and the singlemode operation represents generation of a mode from the a group (Fig. 7, $L_{2}=3$ ). However, at longer $L_{2}$ two types of the single-mode regimes are possible (Fig. 7, $L_{2}=$ $4, \ldots, 7)$. Namely, at relatively small values of parameter $L$ this is generation of a mode from the a group again, whereas the increase of $L$ results in a bifurcation: singlemode operation of a mode becomes unstable and substitutes with stable single-mode operation of $\mathrm{b}$ mode.

One should stress that the possibility for achieving high efficiency in the studied system is provided just due to the 


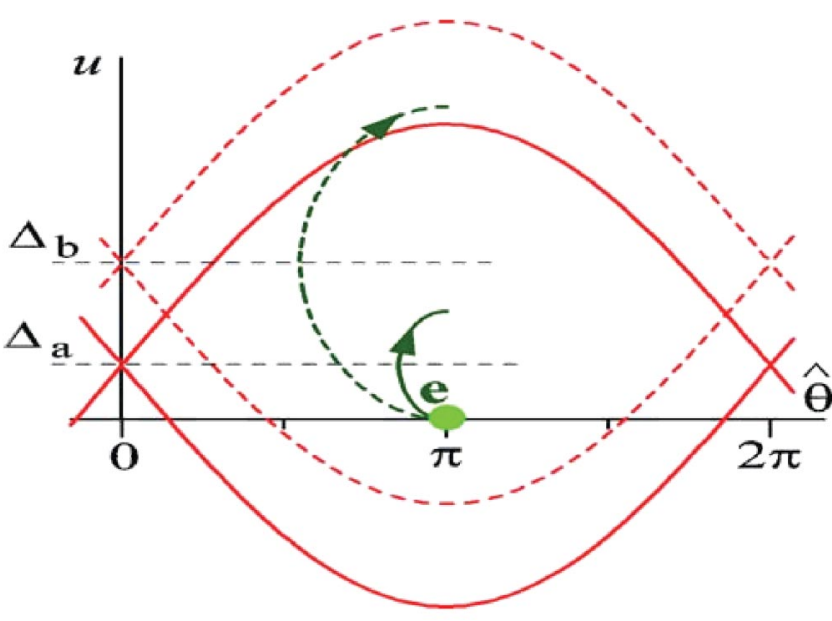

FIG. 10. (Color) Buckets and trajectories of the electron bunch on the phase plane in the cases of a regimes (solid curves) and b regimes (dashed curves).

existence of the effect of "winning" the high-efficiency b mode in its competition with the low-efficiency a mode.

This effect is illustrated in detail in Fig. 11, where the case of $L_{2}=4$ is considered. In the beginning of the process of excitation, both groups of modes take part in the competition, although the a modes grow faster. At $L=$ 5.4, a one mode from the a group wins in the competition. At $L=5.5$ a bifurcation occurs: the transient process becomes two stage. At the first stage $(\tau<10000)$, mainly fast-growing modes from the a group are excited. However, during the second stage $(\tau>10000)$ these modes are suppressed by modes from the $b$ group, and after that the stable single-mode operation of a mode from the $b$ group builds up (a similar effect of transition from generation of a lowefficiency mode to generation of a high-efficiency one was studied in [11]). A similar process of the mode interaction takes place at $L=5.8$; the only difference is a shorter time needed for the $\mathrm{b}$ modes to suppress the a modes.

Certainly, the further increase of $L$ results in the loss of stability of the single-mode operation, and at $L=5.9$ the steady-state process represents generation of several modes from both groups (Fig. 7).

\section{CONCLUSION}

In a FEM oscillator with a broadband feedback system and a three-stage klystronlike interaction region, the amplification band consists of two separate groups of modes,

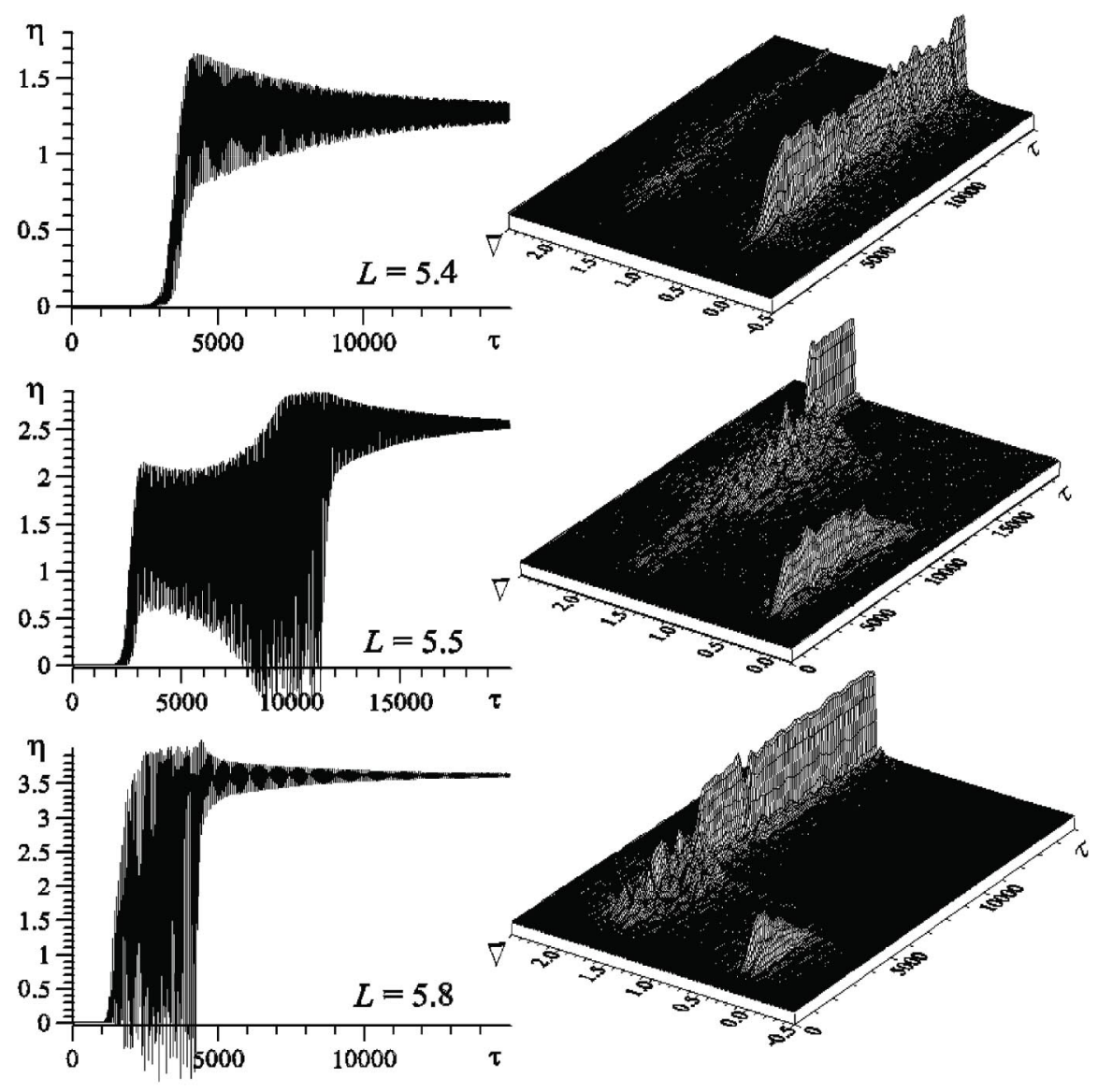

FIG. 11. Spatiotemporal dynamics of excitation of a FEM-oscillator with a klystronlike interaction region $(R=0.5)$. Normalized electronic efficiency, $\eta$, and frequency spectrum of excited modes, $a_{\Delta}$, versus the normalized time, $\tau$, for various lengths of the interaction region, $L$. (the length of the bunching section is $L_{2}=4$ ). 
namely, close-to-resonance modes with high small-signal gains and modes, which are relatively far from the electron-wave resonance and, therefore, have smaller gains of growth from initial noises. As compared to homogeneous systems, the number of competing modes decreased, which simplifies the process of the excitation and improves stability of the single-mode operation. The process of mode interaction represents basically a competition between modes from the two groups described above. At some conditions, a far-from-resonance mode "wins" in the competition, which provides stable single-mode operation regimes with efficiency, which is significantly higher than the efficiency in homogeneous systems.

\section{ACKNOWLEDGMENTS}

This work was supported by the Russian Science Support Foundation and by the Russian Foundation for Basic Research, Grants No 04-02-17118 and No. 05-0216852.

[1] N. A. Vinokurov and A. N. Skrinsky, Report No. INP 7759, Novosibirsk, 1977.
[2] V. N. Litvinenko, B. Burnham, J. M. J. Madey, S. H. Park, and Y. Wu, Nucl. Instrum. Methods Phys. Res., Sect. A 375, 46 (1996).

[3] I. B. Drobyazko, G. N. Kulipanov, V. N. Litvinenko, I. V. Pinayev, V. M. Popik, I. G. Silvestrov, A. N. Skrinsky, A. S. Sokolov, and N. A. Vinokurov, Nucl. Instrum. Methods Phys. Res., Sect. A 282, 424 (1989).

[4] Ya. L. Bogomolov, V. L. Bratman, N. S. Ginzburg, M. I. Petelin, and A.D. Yunakovsky, Opt. Commun. 36, 209 (1981).

[5] T. M. Antonsen, Jr., and B. Levush, Phys. Fluids B 1, 1097 (1989).

[6] N.S. Ginzburg and A. S. Sergeev, Zh. Tekh. Fiz. 61, 133 (1991) [Sov. Phys. Tech. Phys. 36, 665 (1991)].

[7] V.L. Bratman, G. G. Denisov, N. S. Ginzburg, and M. I. Petelin, IEEE J. Quantum Electron. 19, 282 (1983).

[8] R.F. Sincovec and N.K. Madsen, ACM Trans. Math. Softw. 1, 232 (1975).

[9] V. L. Bratman and A. V. Savilov, Nucl. Instrum. Methods Phys. Res., Sect. A 358, 182 (1995).

[10] V. L. Bratman and A. V. Savilov, Zh. Tekh. Fiz. 65, 174 (1995) [Tech. Phys. 40, 209 (1995)].

[11] N.S. Ginzburg, A. V. Elzhov, A. K. Kaminsky, E. A. Perelstein, N. Y. Peskov, S. N. Sedykh, A. P. Sergeev, and A. S. Sergeev, Nucl. Instrum. Methods Phys. Res., Sect. A 483, 225 (2002). 